\title{
Role of Iron-group Metals in the Induced Codeposition of Molybdenum from Aqueous Solution*
}

\author{
By Hisaaki Fukushima**, Tetsuya Akiyama***, \\ Susumu Akagi*** and Kei Higashi**
}

\begin{abstract}
Binary and ternary alloys of molybdenum with iron-group metals were electrodeposited from ammoniacal tartrate baths under galvanostatic conditions. The relationship between the composition of electrodeposited alloys and the cathode current efficiency indicated the two characteristic alloy compositions; the one appeared at a maximum partial current efficiency of molybdenum and the other corresponded to the limiting content of molybdenum. These characteristic alloy compositions were discussed in connection with the mechanism of the induced codeposition of molybdenum based on the electronic structure of the iron-group metals.
\end{abstract}

(Received March 16, 1979)

\section{Introduction}

In the electrodeposition of Mo with irongroup metals, which is termed "induced codeposition" by Brenner ${ }^{(1)}$, the cathodic behavior of individual metals seems to be so irregular that the complicated mechanism of alloy deposition should be reflected. The authors have been studying the mechanism of the induced codeposition of $\mathrm{Mo}$ with $\mathrm{Ni}$, and reported the results suggesting the formation of the partly-reduced Mo oxide and subsequent reduction of this film by atomic hydrogen ${ }^{(2) \sim(4)}$. Although many hypotheses ${ }^{(5)}$ assuming the existence of the intermediate Mo species have been proposed so far, no complete mechanism has been yet available.

In the present work, the relationships between the composition of binary and ternary Mo alloys with iron-group metals and the partial cathode current efficiencies of individual metals were examined in relation to the number of the holes, and hence the number of the unpaired $3 \mathrm{~d}$ electrons, of iron-group metals, and the role of iron-group metals in the induced

* The major part of this paper was originally published in Japanese in J. Japan Inst. Metals, 42 (1978), 980.

** Faculty of Engineering, Kyushu University, Fukuoka 812, Japan.

*** Graduate School, Kyushu University, Fukuoka 812, Japan.

Trans. JIM codeposition of Mo was discussed.

\section{Experimental}

Five types of plating baths containing molybdic acid, metal(II) sulfate ( $\mathrm{Ni}, \mathrm{Co}, \mathrm{Fe}$, $\mathrm{Zn}$ ), potassium sodium tartrate (as a complexing agent) and ammonium hydroxide (for adjusting $\mathrm{pH}$ of the solution to 10.5) were used for the electrodeposition of $(\mathrm{Ni}, \mathrm{Co}$, or $\mathrm{Fe})-$ $\mathrm{Mo}, \mathrm{Ni}-\mathrm{Co}-\mathrm{Mo}$ and $\mathrm{Ni}-\mathrm{Zn}-\mathrm{Mo}$ alloys. In each bath, concentrations of potassium sodium tartrate and total metal ions were kept constant $\left(0.27 \mathrm{kmol} / \mathrm{m}^{3}\right)$. Metal-percentage of Mo, which was defined by Brenner ${ }^{(6)}$ as (weight of metal Mo/total metal weight contained in bath) $\times 100$, was varied in $(\mathrm{Ni}, \mathrm{Co}$, or $\mathrm{Fe})-\mathrm{Mo}$ baths and $\mathrm{Ni}-\mathrm{Co}-\mathrm{Mo}$ bath in which molar ratio of $\mathrm{Ni}$ to $\mathrm{Co}$ was kept constant, 0.26. In the $\mathrm{Ni}-\mathrm{Zn}-\mathrm{Mo}$ bath, the concentration ratio of $\mathrm{Ni}$ to $\mathrm{Zn}$ was varied at the fixed Mo concentrations of $0.014,0.030$ and $0.100 \mathrm{kmol} / \mathrm{m}^{3}$ to give metal-percentages of Mo of 8,17 and 49 , respectively. After the preparation, each solution was deaerated with nitrogen gas for more than $24 \mathrm{~h}$ prior to electrolysis.

A H-section cell of the sealed type contained a platinum anode in the smaller compartment and a $1 \times 2 \mathrm{~cm}^{2}$ copper cathode in the larger compartment which was separated from the anolyte by a sintered glass to minimize contamination by anodic oxidation products.

Electrolysis was carried out galvanostatically

1979 Vol. 20 
at $1 \mathrm{kA} / \mathrm{m}^{2}$, at $30^{\circ} \mathrm{C}$ for $20 \mathrm{~min}$. The deposited metals were dissolved from the cathode with aqua regia and quantitatively analyzed by atomic absorption spectrometry, and the composition of alloys and the cathode current efficiency were calculated.

Further, the empirical confirmation of the surface film was tried. In order to evaluate the film resistance due to the intermediate Mo lower oxide which might be formed during the electrodeposition of Mo alloys with iron-group metals $^{(5)}$, the parameter $\left(R / R_{0}\right) /\left(\rho / \rho_{0}\right)$ was introduced. $R$ and $R_{0}$ were the resistances across the cathode and the tip of the capillary bridge of the reference electrode during the electrodepositions of Mo alloys and iron-group single metals, respectively. $\rho$ and $\rho_{0}$ were the specific resistances of bulk solutions for the alloy and the iron-group single metal depositions, respectively. In the absence of the surface film, and hence any additional resistance except the solution, the parameter $\left(R / R_{0}\right) /$ $\left(\rho / \rho_{0}\right)$ approaches approximately unity when neglecting the change in specific resistance due to the variation of bath composition in the vicinity of the cathode. On the other hand, the parameter is anticipated to exceed unity under the conditions that there exists a significant ohmic contribution due to the surface film to the measured resistance.

Specific resistance of each solution was measured at $30^{\circ} \mathrm{C}$ using a degital conduct meter. $R$ (or $R_{0}$ ) was determined by the interrupter method ${ }^{(7)}$. The constant current pulse of repetition frequency $18.3 \mathrm{~ms}$, duty factor $96.42 \%$ and of current density $1 \mathrm{kA} / \mathrm{m}^{2}$ was applied to the electrodes (the cylindrical platinum cathode of $0.136 \mathrm{~cm}^{2}$ area and the platinum mesh anode), and the cathode potential (vs N.H.E.)-relaxation waveform was observed on the oscilloscope, from which the potential difference between the capillary tip and the electrode surface, i.e., the resistance overvoltage, was read off.

\section{Results}

Figures 1 and 2 showed the effect of metalpercentage of Mo in the bath on the cathode current efficiency and on the composition of

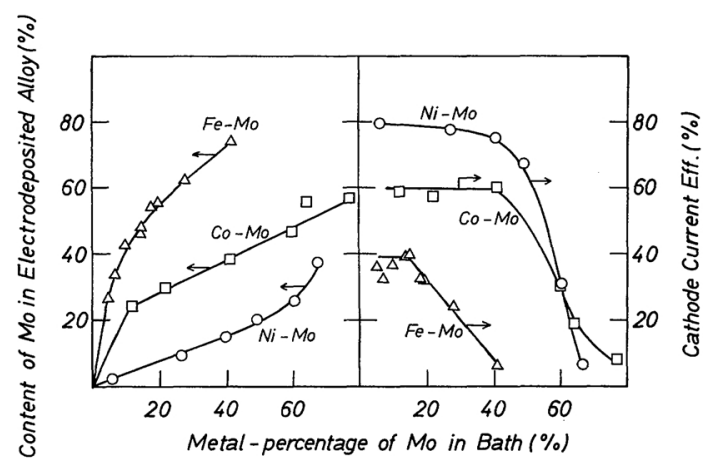

Fig. 1 Effect of metal-percentage of Mo in the bath on the electrodeposited alloy composition and on the cathode current efficiency in the electrodeposition of binary Mo alloys with iron-group metals. Metal-percentage of $\mathrm{Mo}=[\mathrm{wt} . \mathrm{Mo} /$ total wt.(Mo+ iron-group metal)] $\times 100$.

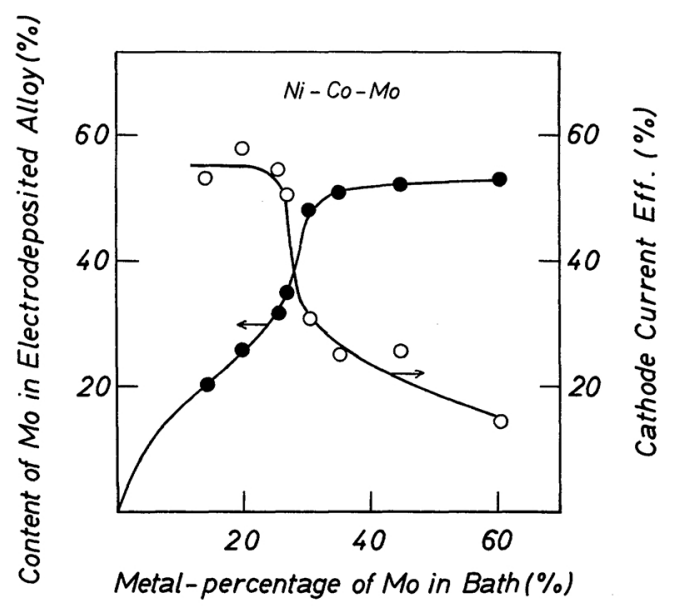

Fig. 2 Effect of metal-percentage of Mo in the bath on the electrodeposited alloy composition and on the cathode current efficiency in the electrodeposition of Ni-Co-Mo alloys.

binary and ternary Mo alloys with iron-group metals, respectively. The Mo content in the electrodeposited alloys increased gradually with increasing the metal-percentage of Mo in the bath. In all cases, a decreasing tendency of the cathode current efficiency for the alloy deposition was recognized with an increase of the Mo content in the alloys. Therefore, the relationship between the Mo content in the alloys and the partial cathode current efficiencies of individual metals were examined, and the results were summarized in Fig. 3. The partial cathode current efficiency of Mo 


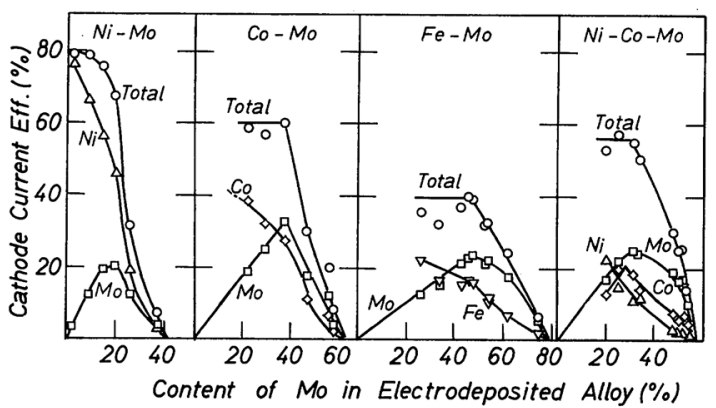

Fig. 3 Relationship between the composition of alloys and the cathode current efficiency during the electrodeposition of Mo-iron-group metal alloys.

was initially increased gradually with the increased Mo content of the alloys, and then decreased to zero after a maximum was achieved. This indicated that it was difficult to obtain the high-Mo content alloy because of its poor current efficiency, and that there existed an upper limit of Mo content. Further, according to Fig. 3, the existence of two characteristic alloy compositions was recognized with respect to the partial cathode current efficiency of Mo; the one appeared at a maximum partial current efficiency of Mo and the other corresponded to the limiting content of Mo.

Figure 4 shows the parameter $\left(R / R_{0}\right) /\left(\rho / \rho_{0}\right)$ measured at regular time intervals from the beginning of the electrodeposition of the alloys from the same baths as for the plating study shown in Fig. 1, plotted as a function of Mo content of the binary alloys. Through an alloy composition range in which the partial cathode current efficiency of Mo showed an increasing tendency in Fig. 3, $\left(R / R_{0}\right) /\left(\rho / \rho_{0}\right)$

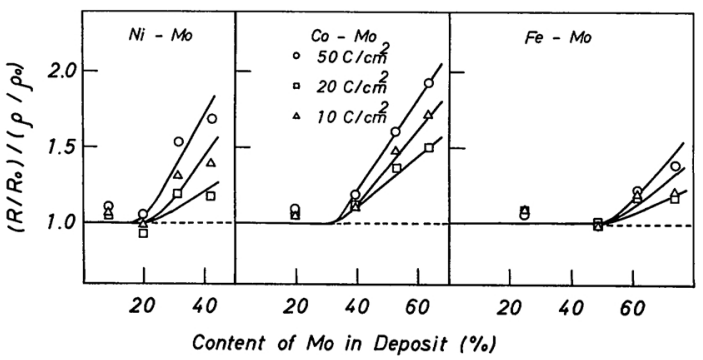

Fig. 4 Relationship between the Mo content in deposit and $\left(R / R_{0}\right) /\left(\rho / \rho_{0}\right)$ in the electrodeposition of binary Mo alloys with iron-group metals at various amount of charge. became approximately unity, indicating the ohmic contribution only due to the solution to the measured resistance overvoltage. On the other hand, $\left(R / R_{0}\right) /\left(\rho / \rho_{0}\right)$ exceeded unity after the Mo content in the alloys at the maximum partial cathode current efficiency of Mo in Fig. 3 was achieved.

\section{Discussion}

1. Speculation of previously proposed mechanisms of the induced codeposition of molybdenum with iron-group metals

Many hypotheses ${ }^{(5)}$ have been proposed so far with respect to the mechanism of induced codeposition of Mo. One of the most plausible mechanisms assumed the formation of an oxide of the partly-reduced reluctant metals such as Mo or W, followed by the reduction of this film by atomic hydrogen. This has been called the catalytic reduction mechanism ${ }^{(8)(9)}$. Our experimental results presented in the previous papers $^{(2) \sim(4)}$ also supported this mechanism. However, this mechanism has been left in an incomplete stage due to the lack of the detailed knowledges of the intermediate compound composition and its formation process. Thus, the mechanism proposed so far represents only an outline of the complicated mechanism of Mo codeposition. Then, the equational expression of this mechanism might be qualitatively given as follows:

$$
\mathrm{MoO}_{4}^{2-}+(6-n) \mathrm{e} \rightarrow \mathrm{Mo}^{n+} \text { oxide (hydroxide) }
$$

$\mathrm{Mo}^{n+}$ oxide (hydroxide) $+n \mathrm{H}(\mathrm{M}) \rightarrow \mathrm{Mo}(\mathrm{M})$

$$
\begin{aligned}
& \mathrm{M}^{2+}+2 \mathrm{e} \rightarrow \mathrm{M} \\
& \mathrm{H}^{+}+\mathrm{e}(\mathrm{M}) \rightarrow \mathrm{H}(\mathrm{M})
\end{aligned}
$$

where $n$ : valence of Mo in the intermediate compound $(0<n<6), \mathrm{M}$ : inducing metal atom, $\mathrm{H}(\mathrm{M})$ : atomic hydrogen held on the inducing metal, $\mathrm{Mo}(\mathrm{M})$ : molybdenum deposited on the inducing metal.

Assuming that the alloy deposition occurs in accordance with the mechanism expressed by the above equations, the cathode current efficiency for the alloy deposition should be 
controlled by the relative depositing rate of $\mathrm{Mo}^{n+}$ oxide and of inducing metal as described later in detail. Therefore, the inducing metal must have following properties in order to gain the alloy deposition with high current efficiency; the high ability of holding atomic hydrogen and the capability of being easily deposited from aqueous solutions. Table 1 showed the heats of atomic hydrogen adsorption on the various metals presented by Delahay et al. ${ }^{(10)}$ The asterisked elements represent the metals which can be electrodeposited from aqueous solutions with high current efficiency reported by Lyons Jr. ${ }^{(11)}$. As is evident from Table 1, only iron-group metals satisfy the above mentioned two properties of the inducing metal. This is in good agreement with the fact that the iron-group metals appeared to be the only alloying elements that make possible the codeposition of substantial amount of reluctant metal.

In the catalytic reduction mechanism ${ }^{(8)(9)}$, the reason why the iron-group metals are the only effective inducing elements is attributed to their electronic structure. Figure 5, quoted from Kittel's work ${ }^{(12)}$, shows the change in the saturation magnetization of $\mathrm{Ni}$ alloys as a function of at. $\%$ of added elements. Since the saturation magnetization in Bohr magnetons per atom corresponds to the unequal distribution of the holes in the two sub-bands, the number of the holes, and hence the number of unpaired $3 d$ electrons, can be seen from Fig. 5. The number of unpaired $3 \mathrm{~d}$ electrons in Fig. 5 is, however, applicable only in the case that an atom exists in the bulk of the metal crystals. In other words, the iron-group metals are predicted by band theory to have the decimal number of unpaired electrons per atom only when they are in a metallic state (form a

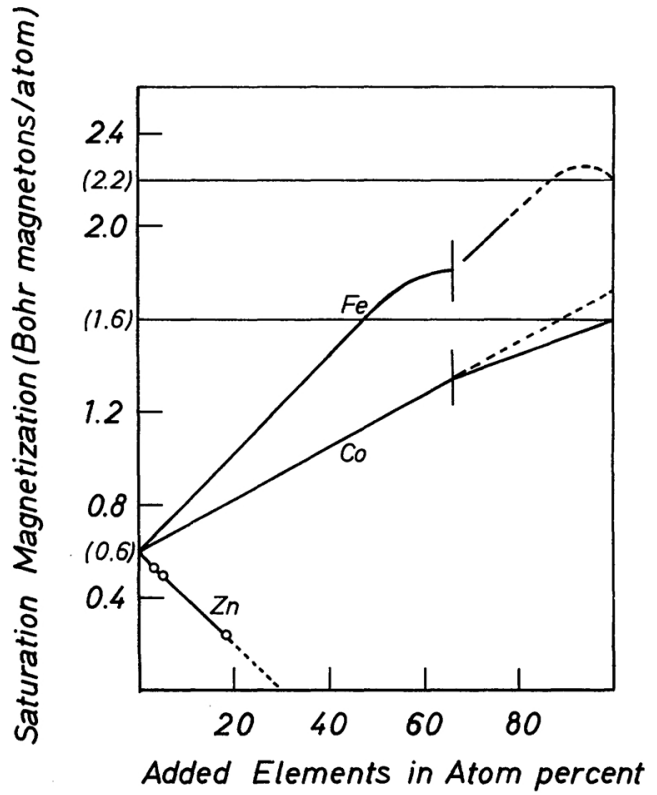

Fig. 5 Saturation magnetization of Ni alloys in Bohr magnetons per atom as a function of the atomic percent of solute element. Quoted from Kittel's work $^{(12)}$.

metallic bond). In the electrodeposition of alloys, however, the inducing iron-group metals no longer exist in the bulk crystal, but in the surface of the electrode. Further, the electronic structure of the iron-group metals may be changed by alloyed Mo, although band theory can not definitely conclude as yet whether the $3 \mathrm{~d}$ band of the iron-group metals is filled with the $5 \mathrm{~s}$ electrons of Mo. As mentioned above, the electronic structure of the iron-group metals in alloy deposition still remains in the incomplete stage. However, since the ferromagnetism in the iron surface has been confirmed recently ${ }^{(13)}$, it is assumed in the following discussion that the electronic structure of

Table 1 Heats of adsorption of atomic hydrogen on the metal $(\mathrm{kJ} / \mathrm{mol})^{(10)}$.

\begin{tabular}{ccccccccc}
\hline \hline $\mathrm{Ag}^{*}$ & $\mathrm{Al}$ & $\mathrm{Au} *$ & $\mathrm{Be}$ & $\mathrm{Cd} *$ & $\mathrm{Cr}$ & $\mathrm{Cu}$ & $\mathrm{Fe}^{*}$ & $\mathrm{Ga}^{*}$ \\
240.6 & 242.7 & 248.2 & 243.6 & 225.6 & 251.5 & 244.8 & 253.2 & 236.5 \\
$\mathrm{Hg} *$ & $\mathrm{In}^{*}$ & $\mathrm{Mg}$ & $\mathrm{Mn}$ & $\mathrm{Mo}$ & $\mathrm{Nb}$ & $\mathrm{Ni}^{*}$ & $\mathrm{~Pb}^{*}$ & $\mathrm{Pd}^{*}$ \\
221.4 & 236.5 & 228.9 & 240.6 & 275.4 & 283.3 & 251.9 & 232.7 & 249.0 \\
$\mathrm{Pt}$ & $\mathrm{Ta}$ & $\mathrm{Ti}$ & $\mathrm{Tl}$ & $\mathrm{V}$ & $\mathrm{W}$ & $\mathrm{Zn} *$ & & \\
263.2 & 287.1 & 255.3 & 231.4 & 258.2 & 287.1 & 229.8 & & \\
\hline \hline
\end{tabular}

* Metal which can be electrodeposited from aqueous solution with high cathode current efficiency ${ }^{(11)}$. 
the iron-group metals which exist in the cathode surface is the same as the one in bulk crystals.

\section{Relationship between the number of} unpaired electrons of iron-group metals and the characteristic alloy compositions

The catalytic reduction mechanism ${ }^{(8)(9)}$ implies that the iron-group metals are in order of the magnitude of the number of their unpaired electrons with respect to the ability of inducing Mo codeposition, since the atomic hydrogen held on the iron-group metals per atom is equal in number to their unpaired electrons. From Fig. 5, the number of unpaired $3 \mathrm{~d}$ electrons of $\mathrm{Ni}, \mathrm{Co}$ and $\mathrm{Fe}$ were 0.6, 1.6 and 2.2, respectively, and the degree of two characteristic compositions shown in Fig. 3 corresponded to the order of the magnitude of the number of unpaired electrons. This suggests that these characteristic compositions are closely related to the unpaired electrons of the iron-group metals.

As pointed out in the discussions about Figs. 3 and 4, the existence of the resistive components on the cathode was confirmed not in the alloy composition range of the increased partial cathode current efficiency of Mo, but in the range of the decreased one. According to the above mechanism, this indicates that although the continuous deposition of Mo can occur while the depositing rate of the irongroup metal is greater enough than that of $\mathrm{Mo}^{n+}$ oxide, the lag in the reduction of $\mathrm{Mo}^{n+}$ oxide occurs with further increase in the formation rate of $\mathrm{Mo}^{n+}$ oxide, permitting the accumulation of unreduced oxide on the cathode. Thus, the partial cathode current efficiency of Mo may be increased by increasing the formation rate of $\mathrm{Mo}^{n+}$ oxide while the reduction of $\mathrm{Mo}^{n+}$ oxide to metal occurs rapidly enough in the presence of freshly deposited iron-group metal. On the other hand, it may be decreased when unreduced $\mathrm{Mo}^{n+}$ oxide is left on the cathode, resulting in the formation of the surface film to make the deposition of the iron-group metals difficult due to the film resistance. Therefore, the maximum partial current efficiency of Mo seems to be attained when the formation rate of $\mathrm{Mo}^{n+}$ oxide is balanced by the depositing rate of the iron-group metal. With regard to one atom of iron-group metals, alternatively, the above situation leads to the gradual increase in the frequency of participation of the unpaired electrons in the reduction of $\mathrm{Mo}^{n+}$ oxide with its increased formation rate. Then, let us consider the situation that the electrodeposition of alloys occurs, in which the unpaired electrons of the iron-group metal participate once in Mo deposition. It requires $n$ hydrogen atoms to reduce one $\mathrm{Mo}^{n+}$ oxide molecule to pure Mo. Therefore, the number of Mo atoms deposited in the presence of one iron-group metal atom having $\mathrm{N}$ unpaired $3 \mathrm{~d}$ electrons is $\mathrm{N} / n$. The weight percentage of Mo in alloy is then given as follows:

$$
\% \text { of } \mathrm{Mo}=[(95.94 \mathrm{~N} / n) /(\mathrm{W}+95.94 \mathrm{~N} / n)] \times 100 \text {, }
$$

where $W$ is an atomic weight of the iron-group metal.

The relationship between the number of unpaired $3 d$ electrons and the Mo content in the electrodeposited alloys was calculated using above eq. The results are shown in Fig. 6. In the calculation, the mean value of the atomic weights of the iron-group metals was used since they do not differ so much from each other, and $n(1,2, \cdots, 6)$ was taken as a parameter. The Mo content observed at the maximum partial current efficiency of Mo in Fig. 3 was plotted against the $\mathrm{N}$ value obtained from Fig. 5. As can be seen from Fig. 6, the Mo content in the alloys at the maximum partial current efficiency of Mo was in good agreement with the curve for $n=4$. The authors $^{(3)(14)}$ have already reported that the cathode current efficiency of $\mathrm{Ni}-\mathrm{Mo}$ alloys was closely related to the depositing rate of Mo lower oxide from the Mo single bath and that the spectroscopic analysis of this hydrated oxide revealed the composition of $\mathrm{MoO}_{2-x}(\mathrm{OH})_{2 x}(x: 0 \sim 2)$. Consequently, the valence of Mo in the intermediate compound is 4 , and the maximum partial current efficiency of Mo appears to be attained when the unpaired electrons of the iron-group metal participates once in the reduction of the Mo intermediate compound.

In case the Mo content of the alloys exceeded 


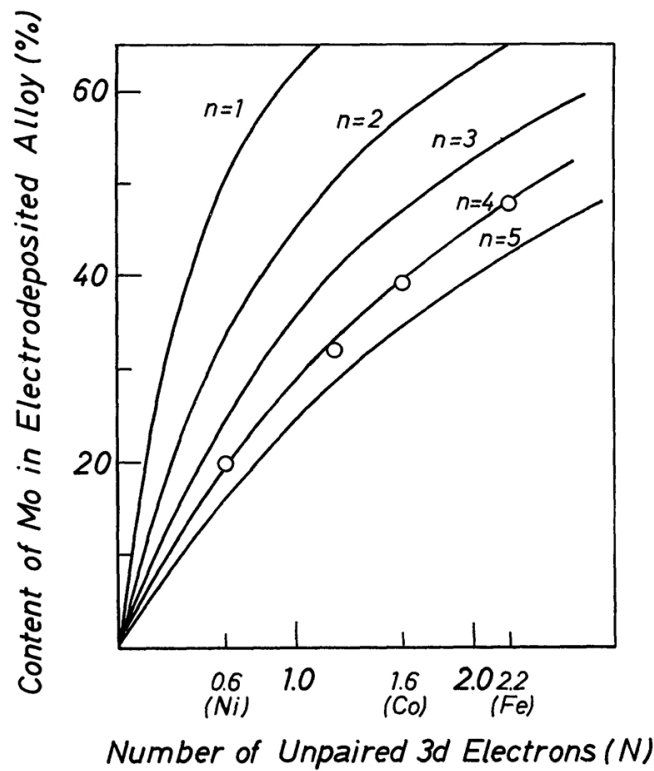

Fig. 6 Relationship between the number of unpaired 3d electrons and the Mo content in deposit during the electrodeposition of Mo with iron-group metals. Solid lines: calculated relationship

$\bigcirc$ : Mo content in deposit observed at the maximum partial cathode current efficiency of Mo

the one at the maximum partial cathode current efficiency of Mo, the participation frequency of the iron-group metal in the reduction of $\mathrm{Mo}^{n+}$ oxide should be more than once. The alloy composition can then be expressed by the following eq. involving $m$, the frequency of participation of the iron-group metal in Mo deposition:

$$
\begin{aligned}
& \% \text { of Mo } \\
& \quad=[(95.94 m \mathrm{~N} / n) /(\mathrm{W}+95.94 m \mathrm{~N} / n)] \times 100 .
\end{aligned}
$$

Figure 7 was obtained by altering the result shown in Fig. 3 into the relationship between the cathode current efficiency and $m$, using the

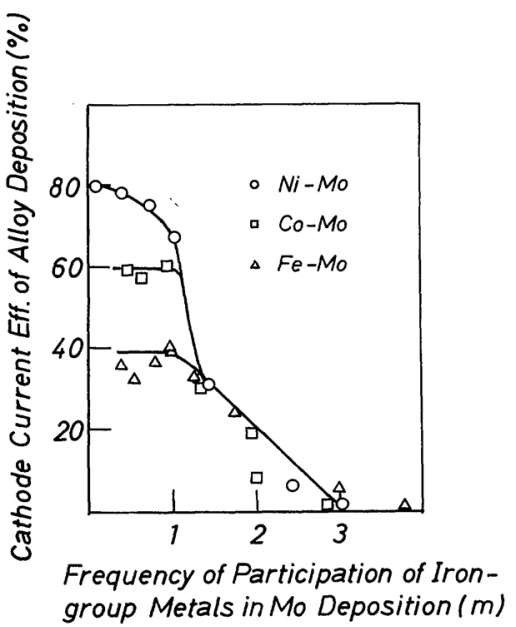

Fig. 7 Relationship between the cathode current efficiency of alloy deposition and the frequency of participation of iron-group metals in Mo deposition.

above eq. for $n=4$. Within the region of $m \leq 1$, the cathode current efficiency for individual iron-group metal deposition was reflected in that of alloy deposition. When $m>1$, i.e., a given iron-group metal atom must participate several times in Mo deposition, the current efficiency tended to be decreasing rapidly to zero at $m=3$. Thus, the maximum frequency of participation of the iron-group metal in Mo deposition seems to be 3 , and the alloy composition at $m=3$ will give the limit of Mo deposition.

Table 2 shows the comparison of the alloy composition at the maximum partial current efficiency of Mo derived from Fig. 3 with that calculated under the assumption that the intermediate $\mathrm{Mo}^{4+}$ oxide was formed and the reduction of this lower oxide film was participated once by the iron-group metals. Table 2 also shows a comparison of the limit-

\begin{tabular}{|c|c|c|c|c|}
\hline \multirow{2}{*}{$\begin{array}{l}\text { Inducing } \\
\text { metal }\end{array}$} & \multicolumn{2}{|c|}{$\begin{array}{l}\text { Cont. of Mo in alloy at the max. } \\
\text { partial C.C.E. of Mo (wt } \%)\end{array}$} & \multicolumn{2}{|c|}{$\begin{array}{l}\text { Limiting content of Mo } \\
\text { in alloy (wt } \% \text { ) }\end{array}$} \\
\hline & experimental. & calculated. & experimental.* & calculated. \\
\hline $\mathrm{Ni}$ & 20.0 & 19.7 & $40 \sim 42$ & 42.4 \\
\hline $\mathrm{Co}$ & 38.0 & 39.4 & $61 \sim 68$ & 66.1 \\
\hline $\mathrm{Fe}$ & 47.5 & 48.6 & 78 & 73.9 \\
\hline $\mathrm{Ni}-\mathrm{Co}$ & 31.7 & 33.8 & $57 \sim 61$ & 61.9 \\
\hline
\end{tabular}

Table 2 Characteristic composition of Mo alloys containing Fe, Co and/or Ni.

* estimated by extrapolation of C.C.E. to zero in Fig. 3. 
ing Mo content estimated by extrapolation of current efficiency to zero in Fig. 3 with the alloy composition calculated assuming 3 times participations of the iron-group metals in Mo deposition. Two characteristic compositions which appeared in the electrodeposition of the iron-group metal-Mo alloys were in good agreement with the calculated ones based on the above assumptions, indicating that the holes in the $3 \mathrm{~d}$ band, and hence the unpaired $3 \mathrm{~d}$ electrons, of the iron-group metals played an essential role in the induced electrodeposition of Mo. This conclusion was also confirmed by the following experiment.

According to Fig. 5, the $3 \mathrm{~d}$ bands of $\mathrm{Ni}$ are filled up with the $4 \mathrm{~s}$ electrons of $\mathrm{Zn}$ in $30 \mathrm{Zn}-$ $70 \mathrm{Ni}$ alloy i.e., the number of unpaired electrons of $\mathrm{Ni}$ is observed to go to zero at about 30 at. $\% \mathrm{Zn}$. Then, the electrodeposition of Mo$\mathrm{Ni}-\mathrm{Zn}$ alloys were carried out. The cathode current efficiencies of Mo and alloy are shown in Fig. 8 as a function of atomic ratio of $\mathrm{Zn}$ to $\mathrm{Ni}$ in the alloys. It is indicated that the cathode current efficiency is decreased to zero at the atomic ratio $\mathrm{Zn} / \mathrm{Ni}$ of about 0.4 , and hence about 30 at. $\% \mathrm{Zn}$, in the deposit.

\section{Summary}

Binary and ternary Mo alloys with iron-group metals were electrodeposited from the ammoniacal tartrate bath under galvanostatic conditions, and the relationships between the

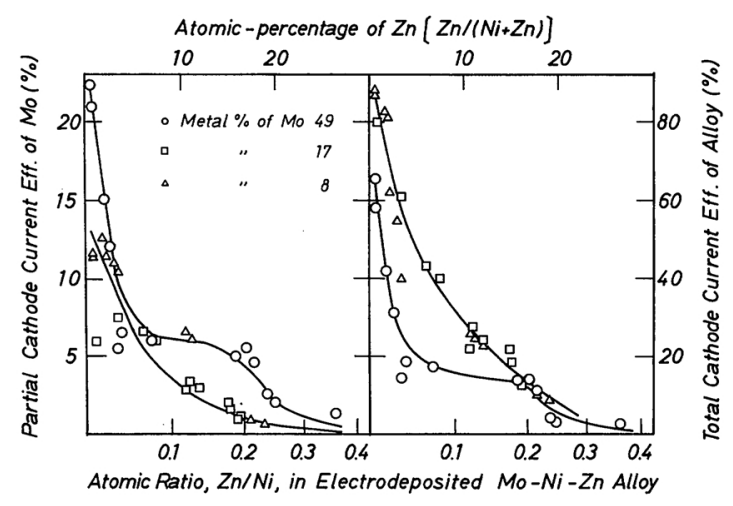

Fig. 8 Relationship between the cathode current efficiency and the atomic ratio of $\mathrm{Zn}$ to $\mathrm{Ni}$ in the electrodeposited Mo-Ni-Zn alloys. composition of electrodeposited alloys and the cathode current efficiency were studied. With increased Mo content of the alloys, the partial current efficiency of Mo tended to increase initialy and then decreased to nearly zero after the maximum was achieved. This showed the existence of two characteristic alloy compositions, the one appeared at a maximum partial current efficiency of Mo and the other corresponded to the limiting Mo content. These characteristic compositions were in good agreement with the calculated ones based on the number of unpaired $3 \mathrm{~d}$ electrons of iron-group metals. Further studies on the electrodeposition of $\mathrm{Ni}-\mathrm{Zn}-\mathrm{Mo}$ alloys indicated that the electrodeposition of Mo ceased when the alloy composition was achieved, in which atomic ratio of $\mathrm{Zn}$ to $\mathrm{Ni}$ corresponded to the one that the positive holes in $3 \mathrm{~d}$ band of $\mathrm{Ni}$ were filled up with $4 \mathrm{~s}$ electrons of $\mathrm{Zn}$. Thus, the unpaired 3d electrons of freshly deposited irongroup metals appeared to play an important role in the induced codeposition of Mo.

\section{REFERENCES}

(1) A. Brenner: Electrodeposition of Alloys 1, 2, Academic press, New York and London, (1963).

(2) K. Higashi and H. Fukushima: J. Metal Finishing Soc. Japan, 24 (1973) 486.

(3) K. Higashi, H. Fukushima, H. Ohashi and T. Akiyama: ibid., 27 (1976), 590.

(4) K. Higashi, H. Fukushima and K. Kitamura: ibid., 28 (1977), 112.

(5) K. Higashi and H. Fukushima: Bull. Japan Inst. Metals, 13 (1974), 865.

(6) Ref. (1), 1, p. 79.

(7) T. Hayashi and T. Ishida: J. Metal Finishing Soc. Japan, 17 (1966), 419.

(8) B. S. R. Sastry: Metal Finishing, 63 [11] (1965), 86.

(9) D. W. Ernst and M. L. Holt: J. Electrochem. Soc., 105 (1958), 686.

(10) P. Rüetschi and P. Delahay: J. Chem. Phys., 23 (1955), 195.

(11) E. H. Lyons, Jr.: J. Electrochem. Soc., 101 (1954), 363.

(12) C. Kittel: Introduction to Solid State Physics, 2nd Ed., John Wiley and Sons, Inc., New York, (1956), p. 335.

(13) T. Shinsyo: Hyomen, 15 (1977), 678.

(14) K. Higashi, H. Fukushima and M. Kanda: J. Met. Inst. Japan, 92 (1976), 561. 